\title{
FORMATION AND EVOLUTION OF CIRCUMSTELLAR AND INTERSTELLAR PAHS: A LABORATORY STUDY
}

\author{
C.S. Contreras ${ }^{1}$, C.L. Ricketts ${ }^{1}$ and F. Salama ${ }^{1}$
}

\begin{abstract}
Studies of dust analogs formed from hydrocarbon $\mathrm{CH}_{4}$, $\mathrm{C}_{2} \mathrm{H}_{2}, \mathrm{C}_{2} \mathrm{H}_{4}, \mathrm{C}_{2} \mathrm{H}_{6}$ ) and PAH precursors have been performed using a new facility that we have developed to simulate interstellar and circumstellar processes. The species formed in a plasma are detected, characterized and monitored in situ with high-sensitivity techniques, which provide both spectroscopic and ion mass information. From these measurements we derive information on the nature, the size and the structure of dust particles, as well as a better understanding of the growth and destruction processes of extraterrestrial dust.
\end{abstract}

\section{Introduction}

The study of the formation, growth and destruction mechanisms of cosmic dust grains is essential for a correct understanding of the physical and chemical evolution of the interstellar medium (ISM). Dust particles span a continuous size distribution from large molecules to nanometer-sized particles to $\mu \mathrm{m}$-sized particles and influence many processes in the evolution of the ISM such as the energy balance through the photoelectric effect, the ionization balance through recombination with electrons and ions, and the chemical composition of molecular clouds (Tielens 2005, see contributions by Verstraete and Bierbaum et al. elsewhere in this volume). The carbonaceous component of cosmic dust plays an important role in the universe (Henning \& Salama 1998). Carbon particles are thought to be primarily formed in the outflow of carbon stars, through a combustion-like process where small carbon chains (e.g., acetylene) form polycyclic aromatic hydrocarbons (PAHs) that nucleate into larger-size PAHs and, ultimately, into nanoparticles (Frenklach et al. 1989; Pascoli \& Polleux 2000; Jäger et al. elsewhere in this volume). According to the model, that was developed to account for the formation of carbonaceous meteoritic materials, nucleation occurs above $2000 \mathrm{~K}$, followed by the growth of amorphous carbon on the condensation nuclei in the 2000-1500 K

1 NASA Ames Research Center, Moffett Field, CA, USA

(C) EAS, EDP Sciences 2011 DOI: $10.1051 /$ eas/1146021 
temperature range. As the temperature falls to around $1100 \mathrm{~K}$, aromatic molecules begin to form in the gas phase and condense onto the growing particles forming graphitic microstructures that will ultimately aggregate into larger structures such as seen in soot formation (Cherchneff et al. elsewhere in this volume).

Although a large amount of laboratory data exists on the formation and the properties of solid dust analogs and in particular of Si-based materials (e.g., Michael et al. 2003), very little is known, about the specific properties (structure, size and spectral signatures) and the formation mechanisms of the key intermediate range of the size distribution (nanoparticles) of cosmic dust. The scarcity of relevant laboratory data on the transition from gas-phase molecules to solid grains is due to the difficulty of forming and isolating these large species and in tracking their evolutionary path under astrophysically realistic laboratory conditions.

Here we describe new laboratory studies that have been performed to address this key issue. First we describe the experimental set-up that has been developed to measure the formation and destruction mechanisms of carbonaceous grains from molecular precursors in an environment with key physical parameters expected to exist in circumstellar regions where molecular formation is observed. We then present and discuss the preliminary results that were obtained in the laboratory and conclude with a discussion of the implications of these new results.

\section{Experimental}

\subsection{Experimental apparatus}

The Cosmic Dust Simulation Chamber (CDSC) was recently set up in our laboratory for the study of cosmic analogs (Fig. 1). The CDSC has been previously used to obtain the first electronic spectra of PAHs and small nanoparticles under conditions that realistically simulate astrophysical environments (for a review see Salama 2008). It combines three complementary techniques: Cavity Ring Down Spectroscopy (CRDS), Pulsed Discharge Nozzle (PDN) Expansion and Reflectron Time-of-Flight Mass Spectrometry (ReTOF-MS) providing both spectroscopic and ion mass information for the identification of the species (large molecules, nanosized grains) that are formed in the plasma source.

The PDN allows the study of molecules, ions and particles isolated in the gas phase. The species are generated in the hot, confined, plasma from carbonaceous precursors and are suddenly frozen by the expansion (Jost 1996) providing a very efficient cooling over short distances (a few $\mathrm{mm}$ ) and an ideal simulation tool for astrophysical environments. The current geometry of the PDN leads to a residence time of a few microseconds for the particles in the active region of the discharge. The plasma expansion generated by the PDN is well characterized (Remy et al. 2003; Broks et al. 2005) and is probed by CRDS several mm downstream with a sub-ppm to ppm sensitivity. CRDS is based on a Nd:YAG pumped tunable pulsed dye laser for the injection of UV-visible photons into a high-finesse cavity (Biennier et al. 2003; Tan \& Salama 2005). For the ReTOF-MS, in order to 


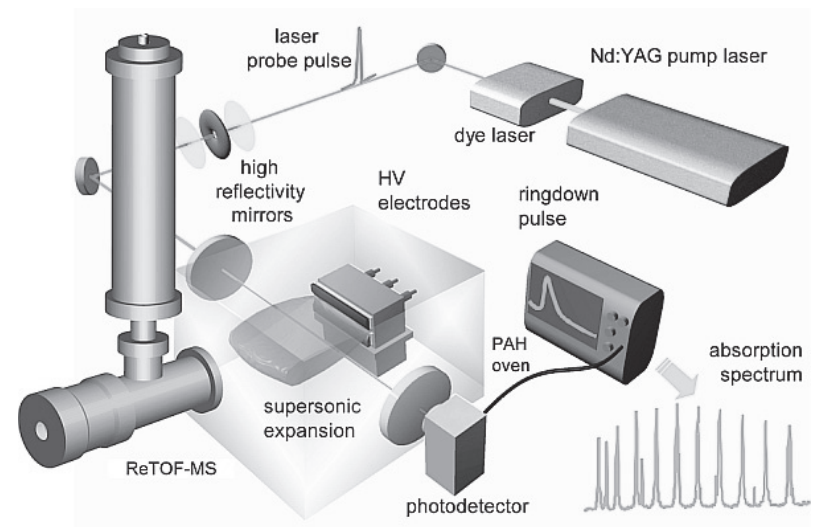

Fig. 1. The Cosmic Dust Simulation Chamber (CDSC) consists of a Pulsed Discharge Nozzle coupled to a Cavity Ring Down Spectrometer apparatus and to an orthogonal, Reflectron time-of-flight mass spectrometer (PDN-CRDS-oReTOFMS).

collect and analyze the ions formed in PDN plasma, a portion of the expansion is skimmed through a $2 \mathrm{~mm}$ orifice which is attached to a long tube that leads to a second aperture of $2 \mathrm{~mm}$. The second aperture is polarized $(-30 \mathrm{~V})$ in order to attract the positive ions from the plasma. A repelling pulse of $+200 \mathrm{~V}$ is applied when the positively-charged ions have arrived in the extraction region; the ions are steered orthogonally to their original flight path, to minimize noise, and guided into the free flight tube of the ReTOF-MS. The ion pulse is then back reflected with the Reflectron optics toward the MCP detector. The ions are dispersed in time according to their velocity (proportional to their mass-to-charge ratio, $\mathrm{m} / \mathrm{z}$ ) so the discrete packets of different $m / z$ ions are detected (Ricketts et al. 2011).

\subsection{Experimental procedures}

The laboratory species that are generated in the expanding discharge plasma experience a strong temperature gradient that ranges from a few thousand degrees Kelvin to $100 \mathrm{~K}$ over a short distance $(1.5-2.5 \mathrm{~mm})$ from the edge of the plasma chamber to the probing zone where the particles are detected (Remy et al. 2003; Broks et al. 2005; Biennier et al. 2006). This temperature domain corresponds to the domain where aromatic molecules begin to form in the gas phase and condense onto the growing particles forming graphitic microstructures that will ultimately aggregate into larger soot-like structures as we observe. This temperature variation is similar to the temperature variation observed from the surface of a carbon star to the edges of the cooling stellar outflow envelope (Pascoli \& Polleux 2000).

Plasma discharges were conducted with small hydrocarbons, methane, ethane, ethylene, and acetylene representative of the alkane, alkene, and alkyne groups, respectively. The formation of larger molecules and carbon grains was probed by the addition of small PAH precursors (naphthalene, 1-methylnaphthalene, 


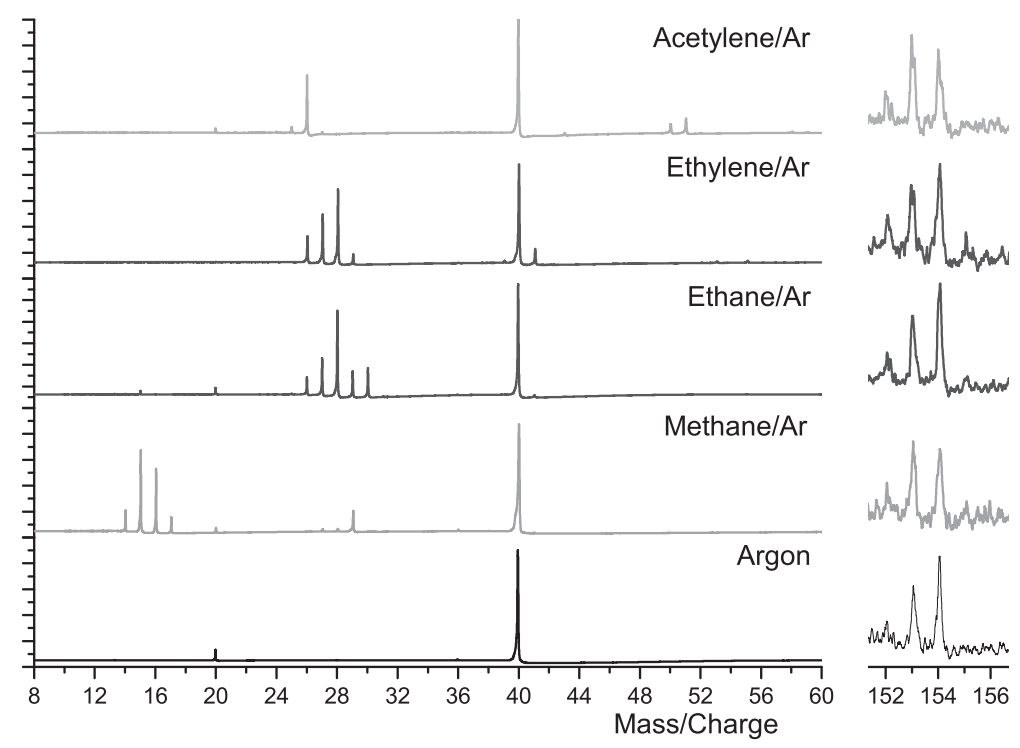

Fig. 2. Representative mass spectra for plasma discharges into Argon seeded with hydrocarbons and/or PAHs. Left: hydrocarbons in Argon; Right: mixtures of Hydrocarbons and Acenaphthene $(154 \mathrm{~m} / \mathrm{z})$ in Argon (close-up of the region for the Acenaphthene ion).

acenaphthene) into the hydrocarbon-seeded Ar gas mixture. In all experiments, gases are seeded into Ar and expanded through the PDN. Solid and liquid PAH samples are held in the bottom reservoir of the PDN and are heated to increase their vapor pressure. In typical experiments, the high voltage discharge is $300 \mu \mathrm{s}$ long and is applied within a gas pulse that lasts $1.2 \mathrm{~ms}$. The ReTOF-MS extraction pulse event is timed to coincide with the discharge and the time it takes the ions to reach the mass spectrometer. The extraction pulse occurred $200 \mu$ s after the discharge.

\section{Preliminary results}

The spectra of the discharge products are shown in Figure 2.

\subsection{Linear hydrocarbon precursors:}

\subsubsection{Alkanes}

In the case of methane, a 1-C alkane, carbon build up leads to structures with 2, 3 and 4 carbons. The highest mass ion detected is $\mathrm{C}_{4} \mathrm{H}_{6}^{+}$. Dehydrogenation (from $\mathrm{CH}_{3}$ to bare $\mathrm{C}$ ) is also observed. Carbon build up is also observed in the case of ethane, a 2-C atom alkane, with structures with 2,3 and 4 carbons. The highest mass ion detected is $\mathrm{C}_{4} H_{7}^{+}$. Dehydrogenation (from $\mathrm{C}_{2} \mathrm{H}_{5}$ to $\mathrm{C}_{2}$ ) as well 
as fragmentation into 1-C structures is observed. These results seem to indicate that the 1-C alkane precursor $\left(\mathrm{CH}_{4}\right)$ exhibits a higher reactivity.

\subsubsection{Comparison of the alkane, alkene, and alkyne hydrocarbon classes}

Ethylene and acetylene, both unsaturated hydrocarbons, show greater reactivity and propensity to form larger mass ions than ethane. In the case of ethylene, structures up to $5-\mathrm{C}$ atoms are detected and the highest mass ion being $\mathrm{C}_{5} \mathrm{H}_{9}^{+}$. In the case of acetylene, structures up to 6 - $\mathrm{C}$ atoms are found with the highest mass ion detected is $C_{6} H_{5}^{+}$. It should be noted that acetylene is routinely stored in acetone and that some of the peaks in the mass spectrum (e.g. 58 and $59 \mathrm{~m} / \mathrm{z}$ ) could be due to oxygen containing hydrocarbons (acetone and protonated acetone ions, respectively). The lower reactivity of the alkane, results in lower mass ions formed in the plasma discharge.

\subsection{Polycyclic aromatic hydrocarbons}

As a basis for the study of the formation of larger PAHs, the naphthalene moiety was used and the reactivity of hydrocarbon-substituted naphthalene was studied. Experiments with naphthalene $\left(\mathrm{C}_{10} \mathrm{H}_{8}\right)$, 1-methylnaphthalene $\left(\mathrm{C}_{11} \mathrm{H}_{10}\right)$ and acenaphthene $\left(\mathrm{C}_{12} \mathrm{H}_{10}\right)$ were performed. While no evidence for bond formation was found, and therefore no ions with a higher $\mathrm{m} / \mathrm{z}$ than the PAH precursors were detected in these preliminary runs, an interesting fragmentation pattern was observed to be distinct for each $\mathrm{PAH}$ molecule. For example, in the case of naphthalene, the formation of the $\mathrm{C}_{8} \mathrm{H}_{6}^{+}$ion corresponds to a loss of $\mathrm{C}_{2} \mathrm{H}_{2}$ from the precursor. The detection of the $\mathrm{C}_{4} \mathrm{H}_{4}^{+}$and its dehydrogenated ions $\left(\mathrm{C}_{4} \mathrm{H}_{3}^{+}, \mathrm{C}_{4} \mathrm{H}_{2}^{+}\right)$demonstrates the loss of $\mathrm{C}_{6} \mathrm{H}_{x}$ fragments, $\mathrm{C}_{6} \mathrm{H}_{4}, \mathrm{C}_{6} \mathrm{H}_{5}$, and $\mathrm{C}_{6} \mathrm{H}_{6}$, respectively. The hydrogen loss observed for naphthalene seems to occur in a sequential manner, since both the $\mathrm{C}_{10} \mathrm{H}_{7}^{+}$and $\mathrm{C}_{10} \mathrm{H}_{6}^{+}$ions are observed. In the case of 1-methylnaphthalene, the fragmentation process seems to be distinct to that of naphthalene. There is a single $\mathrm{H}$ loss $\left(\mathrm{C}_{11} \mathrm{H}_{9}^{+}, 141 \mathrm{~m} / z\right)$, and instead of a sequential loss of hydrogen, no peak is observed at $140 \mathrm{~m} / z$. A corresponding fragment ion $\left(\mathrm{C}_{9} \mathrm{H}_{7}^{+}\right)$is observed at $115 \mathrm{~m} / z$, which is a total loss of $C_{2} H_{3}$. In the acenaphthene spectrum, two clear, sequential hydrogen losses are observed. One possible explanation for this observation is that the hydrogen loss most likely occur at the two adjacent, saturated carbons, thereby forming a double bond between them, with no other fragment ions detected.

\subsection{PAHs with hydrocarbons}

Plasma experiments with both hydrocarbons and PAHs seeded in Argon indicate minimal reactivity between the two carbon containing species. A notable exception is the formation of the doubly-charged $C_{2} H_{3}^{2+}$ ion $(13.5 \mathrm{~m} / z)$, which only appears in experiments with mixed precursors. With a few exceptions, most of the peaks that are detected in this case follow the trend found for the hydrocarbon-only experiments, with similar relative peaks intensities. PAH molecular ion abundances 
(128, 142, $154 \mathrm{~m} / z$ for naphthalene, 1-methylnaphthalene, and acenaphthene, respectively) increase when mixed with the hydrocarbons, as opposed to PAHs in Argon experiments. Follow-up studies are on-going to identify the processes responsible for the observed ionization yields.

\section{Conclusions}

These preliminary experiments have demonstrated that adding a ReTOF-MS to the Cosmic Dust Simulation Chamber (CDSC) constitutes a powerful tool to probe the formation and fragmentation processes of laboratory analogs of interstellar and circumstellar dust. Experiments with hydrocarbon molecule precursors have indicated the formation of larger species through chain growth in all the cases probed so far. The formation of doubly charged ions in the plasma probably contribute to enhancing the reactivity in the plasma chamber. All experiments show fragmentation of the precursors, with losses of $\mathrm{H}, \mathrm{CH}, \mathrm{C}_{2} \mathrm{H}_{2}$, among other fragments specific to each hydrocarbon/PAH combination. The PAHs naphthalene and acenaphthene show subsequent hydrogen loss, with naphthalene dissociating into smaller hydrocarbon fragments. The discharge has been known to induce breakdown of naphthalene in the plasma when the applied voltage on the electrodes is higher than $-500 \mathrm{~V}$ (Remy et al. 2003). For acenaphthene, the loss of two hydrogen atoms most likely occur at the $\mathrm{C}-\mathrm{C}$ bond that is not part of the aromatic network. Each carbon may lose a hydrogen, forming a double bond between the carbons. The resulting end product is the acenaphthylene $\left(\mathrm{C}_{12} \mathrm{H}_{8}^{+}\right)$ion, that is slightly larger than naphthalene. 1-Methylnaphthalene has a total loss of three hydrogens, where two of the hydrogens are lost in one step. The loss of two carbons to form $\mathrm{C}_{9} \mathrm{H}_{7}^{+}$, and no evidence for the naphthalene cation $(127 \mathrm{~m} / \mathrm{z})$ in the mass spectrum, tend to indicate that there is ring opening and fragmentation along the ring. No other fragments are detected, possibly due to the stabilization effect of ring opening. There are indicators that multiply-charged ions are being produced in the plasma discharge as indicated by the detection of the $\mathrm{C}_{2} \mathrm{H}_{3}^{2+}$ $(13.5 \mathrm{~m} / z)$ and $C_{2} H_{5}^{2+}(14.5 \mathrm{~m} / z)$ ions. While $C_{2} H_{5}^{2+}$ is only detected when ethane is present, $\mathrm{C}_{2} \mathrm{H}_{3}^{2+}$ is detected in most cases. These observations seem to point to the fact that the presence of the PAHs may facilitate the formation of the doubly-charged species between ions and neutrals from the hydrocarbon gas. More experiments are being performed to further explore these reactions.

This research was supported by the NASA APRA and Cosmochemistry Programs of the Science Mission Directorate. C.S. Contreras and C.L. Ricketts hold NPP fellowships at NASA-Ames Research Center. The authors wish to acknowledge fruitful discussions with L. Biennier and the outstanding technical support of R. Walker. 


\section{References}

Biennier, L., Benidar, A., \& Salama, F., 2006, Chem. Phys., 326, 445

Biennier, L., Salama, F., Allamandola, L., \& Scherer, J., 2003, J. Chem. Phys., 118, 7863

Broks, B., Brok, W., Remy, J., et al., 2005, Phys. Rev., E., 71, 36409

Frenklach, M., Carmer, C., \& Feigelson, E., 1989, Nature, 339, 196

Henning, T., \& Salama, F., 1998, Science, 282, 2204

Jost, R., 1996, in Low Temperature Molecular Spectroscopy, ed. R. Fausto, 483 (Kluwer Academic Publishers, Dordrecht, NL), 249

Michael, B., Joseph III, A., \& Lilleleht, L., 2003, ApJ, 590, 579

Pascoli, G., \& Polleux, A., 2000, A\&A, 359, 799

Remy, J., Biennier, L., \& Salama, F., 2003, Plasma Sources Sci. Technol., 12, 295

Ricketts, C.L., Contreras, C.S., \& Salama, F., 2011, Int. J. Mass Spectrom. Ion Processes, 300,26

Salama, F., 2008, in Organic Matter in Space, IAU Proceedings, ed. Kwok, \& Sandford (Cambridge University Press), 357, and references therein

Tan, X., \& Salama, F., 2005, J. Chem. Phys., 122, 084318

Tielens, A., 2005, The Physics and Chemistry of the Interstellar Medium (Cambridge Univ Press, Cambridge, UK) 
